\title{
Upfront triple combination therapy in pulmonary arterial hypertension: a pilot study
}

\author{
Olivier Sitbon ${ }^{1,2,3}$, Xavier Jaïs ${ }^{1,2,3}$, Laurent Savale ${ }^{1,2,3}$, Vincent Cottin', \\ Emmanuel Bergot ${ }^{5}$, Elise Artaud Macari ${ }^{1,2,3}$, Hélène Bouvaist ${ }^{6}$, Claire Dauphin ${ }^{7}$, \\ François Picard ${ }^{8}$, Sophie Bulifon ${ }^{1,2,3}$, David Montani ${ }^{1,2,3}$, Marc Humbert ${ }^{1,2,3}$ and \\ Gérald Simonneau ${ }^{1,2,3}$
}

\begin{abstract}
Affiliations: ${ }^{1}$ Univ. Paris-Sud, Faculté de Médecine, Le Kremlin-Bicêtre, ${ }^{2} A P H P$, Centre de Référence de l'Hypertension Pulmonaire Sévère, Service de Pneumologie et Soins Intensifs, DHU Thorax Innovation, Hôpital de Bicêtre, Le Kremlin-Bicêtre, ${ }^{3}$ INSERM U999, LabEx LERMIT, Centre Chirurgical Marie-Lannelongue, Le Plessis-Robinson, «Univ. Lyon-1, Hospices Civils de Lyon, Centre de Référence des maladies pulmonaires rares, Centre de Compétences de l'Hypertension Pulmonaire, Hôpital Louis Pradel, Lyon, ${ }^{5}$ Service de Pneumologie et d'oncologie thoracique, Hôpital Universitaire de Caen, Caen, ${ }^{6}$ Service de Cardiologie, CHU, Grenoble, ${ }^{7}$ Service de Cardiologie, CHU Gabriel-Montpied, Clermont-Ferrand, and ${ }^{8}$ Service de Cardiologie, Hôpital Haut-Lévêque, Pessac, France.
\end{abstract}

Correspondence: 0. Sitbon, Service de Pneumologie et Soins Intensifs, CHU de Bicêtre, 78 rue du Général Leclerc, F-94275 Le Kremlin-Bicêtre, France. E-mail: olivier.sitbonlabct.aphp.fr

ABSTRACT Patients with severe pulmonary arterial hypertension (PAH) in New York Heart Association (NYHA) functional class (FC) III/IV have a poor prognosis, despite survival benefits being demonstrated with intravenous epoprostenol. In this pilot study, the efficacy and safety of a triple combination therapy regimen in patients with severe PAH was investigated.

Data from newly diagnosed NYHA FC III/IV PAH patients $(n=19)$ initiated on upfront triple combination therapy (intravenous epoprostenol, bosentan and sildenafil) were collected retrospectively from a prospective registry.

Significant improvements in 6-min walk distance and haemodynamics were observed after 4 months' triple combination therapy in 18 patients $(\mathrm{p}<0.01)$; 17 patients had improved to NYHA FC I or II. One patient was not included in the month 4 assessment (due to an emergency lung transplant in month 3 ). At the final evaluation (mean \pm SD $32 \pm 19$ months), all 18 patients had sustained clinical and haemodynamic improvement. Overall survival estimates for the triple combination cohort were $100 \%$ at 1,2 and 3 years. Expected survival calculated from the French equation was 75\% (95\% CI 68-82\%), 60\% (95\% CI 50-70\%) and $49 \%(95 \%$ CI $38-60 \%)$ at 1, 2 and 3 years, respectively.

This pilot study provides preliminary evidence of the long-term benefits of upfront triple combination therapy in patients with severe PAH.

O @ERSpublications

Upfront triple combination therapy shows promising long-term efficacy in severe pulmonary arterial hypertension http://ow.ly/tKgHX

For editorial comments see page 1556.

Received: July 092013 | Accepted after revision: Feb 112014 | First published online: March 132014

Support statement: Editorial support was provided by L. Quine (Elements Communications, Westerham, UK) and funded by Actelion Pharmaceuticals Ltd (Allschwil, Switzerland).

Conflict of interest: Disclosures can be found alongside the online version of this article at www.erj.ersjournals.com 


\section{Introduction}

Pulmonary arterial hypertension (PAH) is a progressive and devastating disease characterised by a progressive increase in pulmonary vascular resistance (PVR). The complex pathogenesis of PAH involves dysfunction of three key pathways: the endothelin pathway, the prostacyclin pathway and the nitric oxide pathway [1]. This dysfunction results in increased cellular proliferation, remodelling of the pulmonary vasculature and vasoconstriction, which leads increased PVR and ultimately right heart failure and death [1]. In the modern treatment era, better understanding of the pathophysiology of PAH and the availability of PAH-specific therapies have improved survival of PAH patients; however, despite these advances, $\mathrm{PAH}$ remains incurable [2].

PAH severity is classified according to guidance provided by the New York Heart Association (NYHA), which links the symptoms of PAH with activity limitation and ranges from functional class (FC) I, where symptoms are minimal and do not limit physical activity, to NYHA FC IV, where symptoms are severe and result in an inability to carry out any physical activity [3]. Current guidelines for the management of PAH recommend that prostacyclins, such as epoprostenol, as first-line therapy in patients in FC III or IV [3]. Although epoprostenol has been shown to improve survival in patients with idiopathic PAH in a randomised control study [4], patients in NYHA FC IV, and those in FC III with a poor cardiac output, still have an extremely poor prognosis [2, 5-7].

Initiation of combination therapy, where an additional PAH-specific therapy is added to background medication, is recommended for patients not responding adequately to monotherapy [3]. However, this sequential approach to combining established PAH therapies has had limited success [8-13]. Additionally, the results of randomised controlled trials with sequential combination therapy were not uniform, with the primary end-point (which was the change in 6-min walk distance (6MWD) in all of these trials) being met in only two out of five studies [8-10, 12, 13].

It has been postulated that upfront combination therapy targeting the different pathways involved in the pathogenesis of PAH may be beneficial to patients with NYHA FC III/IV, and this has been studied in a randomised controlled trial and a recent observational study, with some improvements in clinical characteristics being reported $[14,15]$.

The current study investigated the efficacy of a triple combination regimen consisting of epoprostenol, bosentan and sildenafil in PAH patients in NYHA FC III/IV with severe haemodynamic impairment, and also determined whether triple combination therapy provides enhanced survival benefits in patients with severe PAH.

\section{Methods}

This retrospective study complied with the Declaration of Helsinki. Although French law does not require ethics committee approval or informed consent for retrospective data collection, the data were anonymised and complied according to the requirements of the Commission Nationale Informatique et Liberté, the organisation dedicated to privacy, information technology and civil rights in France. The committee approved the methods used to collect and analyse data on May 24, 2003 (approval number 842063).

\section{Study design}

In this retrospective analysis, data from consecutive newly diagnosed $\mathrm{PAH}$ patients (incident cases) with severe symptoms admitted to the French reference centre and some regional centres for evaluation of PAH, and initiated on upfront triple combination therapy consisting of epoprostenol (intravenous), bosentan and sildenafil were collected. Patients meeting the inclusion criteria were enrolled between December 2007 and July 2012. The observational period ended in July 2013, 1 year after enrolment of the final patient.

\section{Patients}

Adult patients (18-65 years of age) with idiopathic, heritable or anorexigen-associated PAH in NYHA FC III/IV and with severe haemodynamic impairment were eligible for triple combination therapy and included in the study. Severe haemodynamic impairment was defined as cardiac index $<2.0 \mathrm{~L} \cdot \mathrm{min}^{-1} \cdot \mathrm{m}^{-2}$ and/or mean right atrial pressure $>20 \mathrm{mmHg}$ and/or PVR $\geqslant 1000 \mathrm{dyn} \cdot \mathrm{s} \cdot \mathrm{cm}^{-5}$.

Patients with PAH associated with connective tissue diseases, congenital heart disease, HIV infection or portal hypertension were excluded. Patients with non-group 1 pulmonary hypertension were also excluded. 


\section{Treatment regimen}

Epoprostenol

Epoprostenol was infused continuously using a portable infusion pump (CADD-Plus Legacy, Sims Deltec Inc, St Paul, MN, USA). Venous access for the infusion of epoprostenol was obtained by insertion of a permanent, tunnelled Groshong catheter (Bard Access System, Salt Lake City, UT, USA) into a subclavian vein. Epoprostenol therapy $\left(1 \mathrm{ng} \cdot \mathrm{kg}^{-1} \cdot \mathrm{min}^{-1}\right)$ was initiated while patients were in-patients at the expert centre and the dose increased every $12 \mathrm{~h}$. On day 5, once a dose of $10 \mathrm{ng} \cdot \mathrm{kg}^{-1} \cdot \mathrm{min}^{-1}$ had been attained, the patient was discharged. The dose was further up-titrated by $1 \mathrm{ng} \cdot \mathrm{kg}^{-1} \cdot \mathrm{min}^{-1}$ every 2 weeks to a maximum of $16 \mathrm{ng} \cdot \mathrm{kg}^{-1} \cdot \mathrm{min}^{-1}$, depending on the patient's tolerance.

\section{Bosentan}

Bosentan was started concomitantly with epoprostenol therapy at a dose of $62.5 \mathrm{mg}$ twice daily. After 4 weeks, this was increased to $125 \mathrm{mg}$ twice daily for the remainder of the study. Liver function tests were performed every 2 weeks during the first 2 months and monthly thereafter. If liver enzymes were elevated (more than three times the upper limit of normal (ULN)), then bosentan was stopped or the dose adjusted accordingly.

\section{Sildenafil}

Sildenafil was started on study day 5 , at the end of the first epoprostenol titration period. Sildenafil was initiated and maintained at a dose of $20 \mathrm{mg}$ thrice daily.

\section{Study assessments}

Variables assessed at baseline, 4 months after initiation of triple combination therapy and once each year until the study end included NYHA FC, 6MWD and pulmonary haemodynamics (assessed by right heart catheterisation (RHC)). An acute pulmonary vasoreactivity test with inhaled nitric oxide was performed in all patients at the time of the first haemodynamic evaluation. If patients were unable to perform the 6-min walk test at baseline or at any of the follow-up visits, a 6MWD of zero was recorded. The final visit was defined as the time point where a complete evaluation (including RHC) had been conducted. Adverse events were recorded throughout the study period.

\section{Statistical analysis}

Data were stored in a personal computer-based data spreadsheet. Analysis was performed using the StatEL statistical package in Microsoft Excel 2007 (Ad Science, Paris, France). All data are expressed as mean \pm SD. Comparison of 6MWD and haemodynamic variables obtained at baseline, month 4 and final evaluation visits was made using one-way ANOVA for paired groups for normally distributed variables and the nonparametric Friedman test for the non-normally distributed variables. Post hoc comparisons were made using Newman and Keuls test or nonparametric Wilcoxon test, as appropriate. The Chi-squared test for independence was used to compare differences between NYHA FC assessed at the baseline, month 4 and final evaluation visits. A p-value $<0.05$ was considered statistically significant.

Analysis of overall survival was performed using an intention-to-treat approach. For the survival analysis, the date of diagnostic RHC ( $<1$ week before commencement of the treatment) was used as the start point to determine length of survival. The cut-off date was July 31, 2013. The Kaplan-Meier method was used to estimate overall survival at each interval. Patients who underwent lung transplantation were considered as censored at the date of transplantation. Expected survival was calculated using a survival prediction equation (commonly referred as the French equation) using three variables, sex, 6MWD at diagnosis and cardiac output at diagnosis, as previously described [16].

\section{Results}

\section{Baseline patient characteristics}

During the study period, 19 patients were identified as having received the upfront triple combination treatment regimen and included in the analysis. Two additional patients were also considered eligible to receive this treatment regimen but died before therapy was started.

Baseline demographics, exercise capacity and haemodynamics are shown in table 1 . Of the 13 patients who underwent genetic testing, 10 were bone morphogenetic protein receptor type II (BMPR2) mutation carriers and were considered as having heritable PAH. Six patients were unable to perform the 6-min walk test at baseline. No patients demonstrated a positive response to acute pulmonary vasoreactivity testing using current criteria: mean pulmonary arterial pressure (mPAP) decreased from $67.7 \pm 15.8 \mathrm{mmHg}$ (range 47-103 $\mathrm{mmHg}$ ) to $66.1 \pm 17.5 \mathrm{mmHg}$ (range $45-100 \mathrm{mmHg}$ ). 


\section{TABLE 1 Patients' baseline characteristics}

$\begin{array}{lc}\text { Subjects } & 19 \\ \text { Age years (range) } & 39.4 \pm 14.2(18.1-63.1) \\ \text { Females } & 17(89) \\ \text { Idiopathic/heritable/anorexigen-associated PAH } & 9 / 10 / 0 \\ \text { BMPR2 mutation carrier } \mathbf{n} / \mathbf{n} \text { tested (\%) } & 10 / 13(77) \\ \text { NYHA functional class III/IV } & 8(42) / 11(58) \\ \text { 6-min walk distance } \mathbf{m} & 215 \pm 174 \\ \text { Haemodynamics } & \\ \text { Right atrial pressure } \mathrm{mmHg} & 12.2 \pm 5.2 \\ \text { Mean pulmonary arterial pressure } \mathrm{mmHg} & 67.7 \pm 15.8 \\ \text { Pulmonary capillary wedge pressure } \mathrm{mmHg} & 8.3 \pm 3.4 \\ \text { Cardiac output L·min }{ }^{-1} & 2.83 \pm 0.77 \\ \text { Cardiac index L·min }{ }^{-1} \cdot \mathrm{m}^{-2} & 1.64 \pm 0.34 \\ \text { Pulmonary vascular resistance dyn } \cdot \mathrm{s} \cdot \mathrm{cm}^{-5} & 1807 \pm 722 \\ \text { Mean blood pressure } \mathrm{mmHg} & 91.7 \pm 12.2 \\ \text { Heart rate beats per min } & 92.3 \pm 10.7 \\ \text { Mixed venous oxygen saturation } \% & 50.1 \pm 9.0\end{array}$

Data are presented as $\mathrm{n}$, mean $\pm \mathrm{SD}$ or $\mathrm{n}(\%)$, unless otherwise stated. $\mathrm{PAH}$ : pulmonary arterial hypertension; BMPR2: bone morphogenetic protein receptor type II; NYHA: New York Heart Association.

\section{Treatment dosing}

The mean doses of epoprostenol at baseline, month 4 and the final visit are shown in table 2. Adjustment of bosentan dose during the study was not required by any of the patients, although bosentan therapy was withdrawn after 11.5 and 32 months in two patients due to liver enzyme elevation.

\section{Month 4 and long-term follow-up assessments}

Of the 19 patients who initiated the up-front triple combination regimen, 18 experienced significant clinical and haemodynamic improvements after 4 months' therapy $(\mathrm{p}<0.01)$ and 17 were in NYHA FC I/II (fig. 1 and table 2). One patient initiated on upfront triple combination therapy was not included in the month 4 assessment. This 20-year old female with idiopathic PAH and severe clinical and haemodynamic impairment at baseline (NYHA FC IV, 6MWD $0 \mathrm{~m}$; mPAP $103 \mathrm{mmHg}$, cardiac index $1.40 \mathrm{~L} \cdot \mathrm{min}^{-1} \cdot \mathrm{m}^{-2}$ and PVR 3404 dyn $\cdot s \cdot \mathrm{cm}^{-5}$ ) did not improve on therapy and successfully underwent an urgent heart-lung transplantation at month 3 .

All 18 patients assessed at month 4 were followed up (follow-up time: mean $41.2 \pm 13.4$ months; median 39.2 months; range 13.7-69.4 months). The final complete evaluation, including RHC, was performed after

TABLE 2 Functional class, 6-min walk distance (6MWD) and haemodynamics at the baseline, month 4 and final follow-up visits

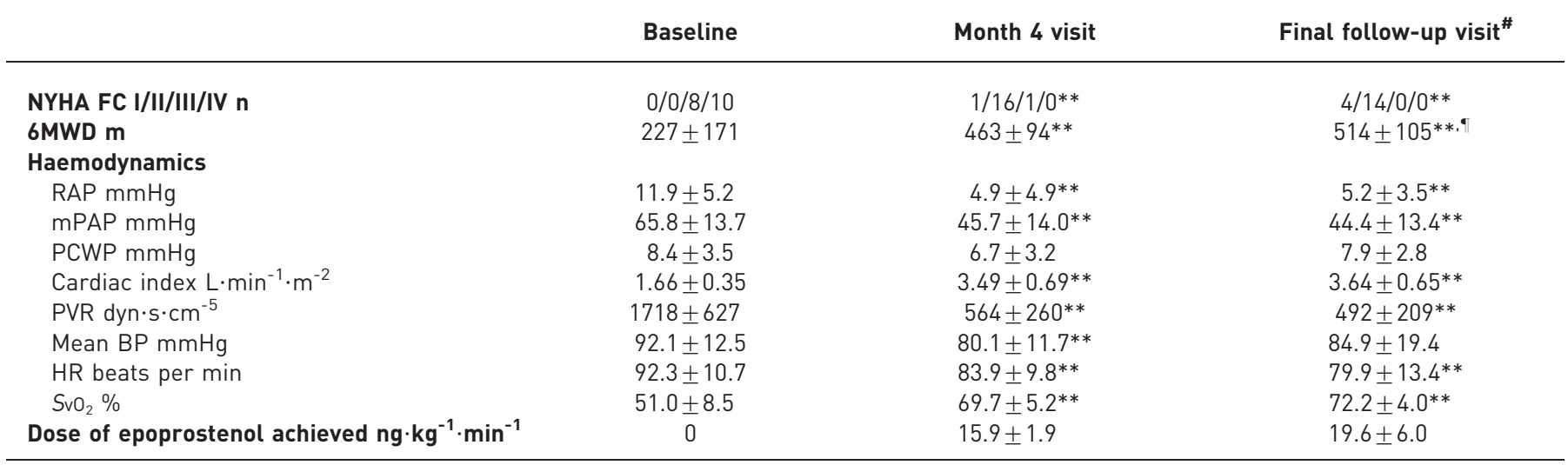

Data are presented as mean \pm SD unless otherwise stated. $n=18$. NYHA: New York Heart Association; FC: functional class; 6MWD: 6-minute walk distance; RAP: right atrial pressure; mPAP: mean pulmonary arterial pressure; PCWP: pulmonary capillary wedge pressure; PVR: pulmonary vascular resistance; $\mathrm{BP}$ : blood pressure; HR: heart rate; $\mathrm{SvO}_{2}$ : mixed venous oxygen saturation. ${ }^{\#}$ : follow-up time $32 \pm 19$ months. ${ }^{* *}$ : $\mathrm{p}<0.01$ versus baseline; ${ }^{\bullet} \mathrm{p}<0.01$ versus month 4 visit. 

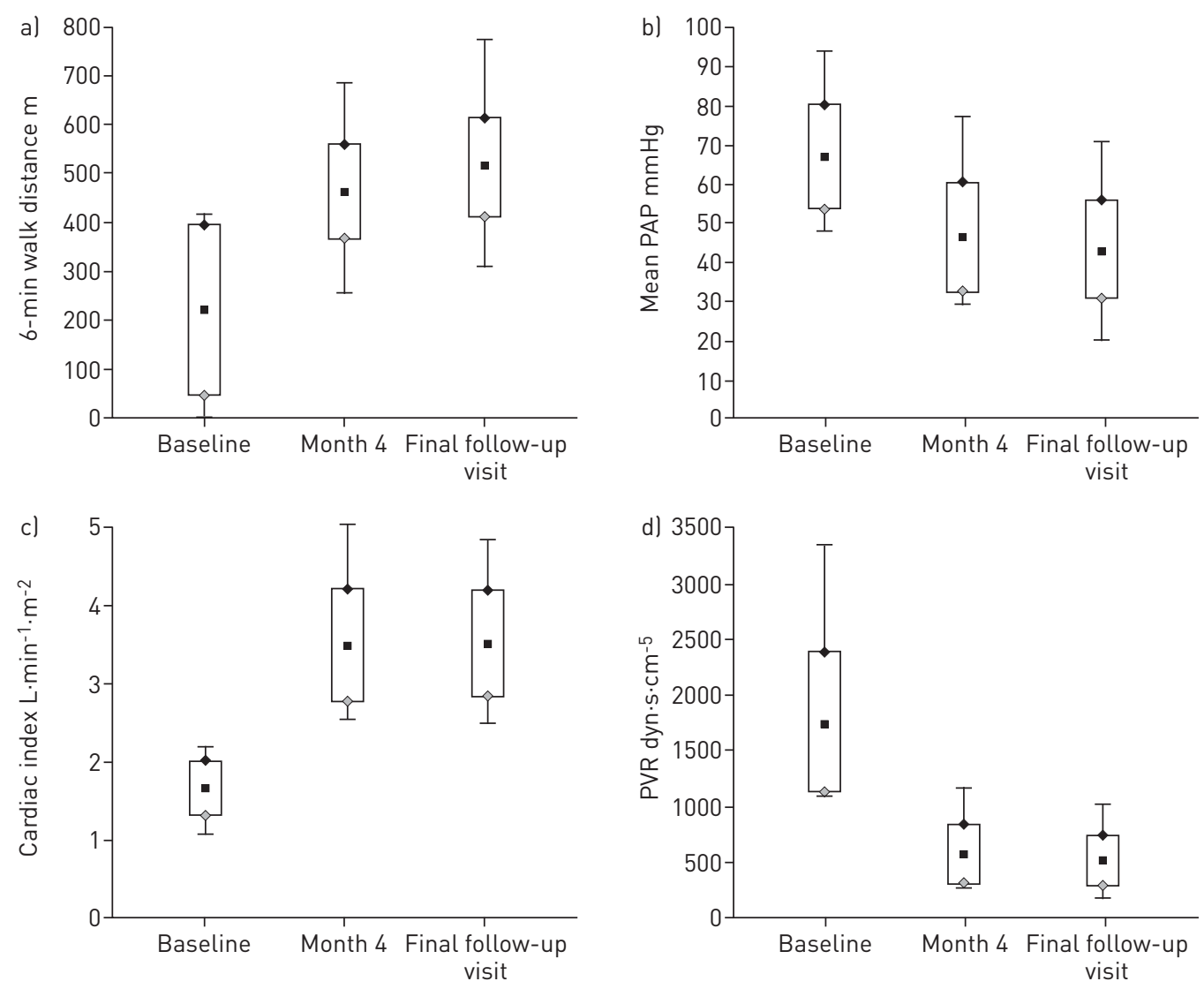

FIGURE 1 Box and whisker plots of a) 6-min walk distance, b) mean pulmonary arterial pressure (PAP), c) cardiac index and d) pulmonary vascular resistance (PVR) at baseline, month 4 and the final follow-up visit (mean \pm sD follow-up time $32 \pm 19$ months) after initiation of upfront epoprostenol, bosentan and sildenafil combination therapy $(\mathrm{n}=18)$. Squares represents the mean, boxes and diamonds represent the standard deviation, and whiskers represent the range.

$32.3 \pm 19.4$ months (range 8.5-73.3 months). Two patients discontinued bosentan due to liver enzyme elevation, but continued on epoprostenol and sildenafil dual therapy. Changes in NYHA FC, 6MWD and haemodynamics during treatment are shown in table 2. All patients had a sustained clinical and haemodynamic improvement. A significant increase in 6MWD was observed between month 4 and the final follow-up visit $(\mathrm{p}<0.01)$. As of September 1, 2013, all 18 patients were alive and in NYHA FC I/II.

\section{Survival analysis}

All patients initiated with upfront triple combination therapy were still alive after a mean follow up of $41.2 \pm 13.4$ months. Overall survival estimates were $100 \%, 100 \%$ and $100 \%$ at 1,2 and 3 years, and respective transplant-free survival estimates were $94 \%, 94 \%$ and $94 \%$. Expected survival calculated from the French equation was 75\% (95\% CI 68-82\%), 60\% (95\% CI 50-70\%) and 49\% (95\% CI 38-60\%) at 1, 2 and 3 years, respectively.

\section{Safety}

Most adverse events were typical of epoprostenol therapy (jaw pain, manageable headache, diarrhoea or flushing). Two patients experienced asymptomatic liver enzyme elevation (more than eight times the ULN), needing bosentan withdrawal after 11.5 and 31.5 months on triple combination therapy. No clinical or haemodynamic deterioration was observed after a follow-up of 32 and 17.5 month in these patients.

\section{Discussion}

Our study describes an alternative treatment strategy to that currently recommended [3] for patients with severe idiopathic and heritable PAH. Patients who received upfront triple combination therapy with epoprostenol, bosentan and sildenafil experienced improvements in FC, exercise capacity and cardiopulmonary haemodynamics. Furthermore, the survival prospects were better in these patients on 
upfront triple combination therapy than could have been expected based on the equation derived from the French Registry.

The patient population evaluated in the current study represents an incident population with particularly severe PAH as evidenced by the high proportion of patients in NYHA FC IV; the degree of their haemodynamic impairment, in particular their very low cardiac output; and the high proportion of patients with the BMPR2 mutation, which has been shown to be associated with more severe haemodynamic compromise at diagnosis $[17,18]$. With the exception of one patient, all those who were treated with triple combination therapy improved from NYHA FC III/IV to at least FC II after 4 months of treatment and this functional improvement was sustained during long-term follow-up. Achieving NYHA FC II following the initiation of therapy has been shown to be an indicator of better prognosis in patients with PAH and, as such, is considered to be a treatment target for patients with $\mathrm{PAH}[3,5,7]$. In addition, initial improvements in haemodynamic variables were maintained to the final visit (mean 32 months) with a further significant increase in 6MWD from month 4 to the final visit being reported. Although two patients discontinued bosentan during the long-term follow-up phase, they were maintained in FC II or lower using a combined regimen of epoprostenol and sildenafil.

The haemodynamic improvements of an increase in cardiac index and a fall in PVR reported in these patients probably contributed to the observed survival benefit following treatment with triple combination therapy. These haemodynamic indices have previously been identified as independent prognostic factors in $\mathrm{PAH}[5,7,19,20]$. It is interesting to note that pulmonary pressures also fell significantly (by an average of $>20 \mathrm{mmHg}$ after 4 months) with upfront triple combination therapy, which is very unusual with all other PAH-targeted therapy regimens. For PAH patients who respond to vasodilators acutely and receive calcium channel blockers, a fall in mPAP associated with a marked increase in cardiac output/index is strongly associated with a long-term survival [21]. One may postulate that observation of this type of haemodynamic response with $\mathrm{PAH}$-targeted therapies may also result in a survival benefit.

A recent study of a similar cohort of patients by KEMP et al. [15] showed that upfront dual therapy with bosentan and epoprostenol improved patients' haemodynamics, FC, exercise capacity and clinical status at month 4. They found that after 4 months' treatment, PVR was significantly improved in patients on dual therapy $(-48 \pm 17 \%)$ compared with patients on epoprostenol monotherapy $(-29 \pm 17 \%)$. Although it is not appropriate to compare results from different studies directly, these data appear to be consistent with the triple combination therapy analysis presented here; change in PVR from baseline to month 4 was $-67 \pm 10 \%$. With regard to overall survival estimates, not all patients in the study by KEMP et al. [15] achieved the same level of treatment success reported here with upfront triple combination therapy. Specifically, overall survival estimates for upfront epoprostenol and bosentan combination therapy were $100 \%, 94 \%, 94 \%$ and $74 \%$ at $1,2,3$ and 4 years, respectively, compared with $87 \%, 77 \%, 67 \%$ and $62 \%$ in the epoprostenol monotherapy group in the study by KeMP et al. [15]; this represented only a trend in overall survival. There was no significant difference in the rate of transplant-free survival, with three patients in the dual therapy group requiring lung transplantation within the first 34 months of therapy [15]. In the study by KEMP et al. [15], analysis of the baseline characteristics showed that the patients who failed on dual therapy had worse baseline values than patients who were treated successfully. Interestingly, the baseline characteristics of patients in the current study were, in general, similar to the patients not benefiting from upfront dual combination therapy in the study by KEMP et al. [15].

In the current analysis of a relatively small number of patients, the greater proportion of whom had more severe disease, survival with triple combination therapy was $100 \%$ at 3 years. In addition, only one of the 19 patients who received triple combination therapy did not improve at month 4 and required heart-lung transplantation; as described in the Results section, she had severe clinical and haemodynamic impairment at baseline. These findings are particularly relevant given that, despite increasing awareness, the majority of PAH patients are in NYHA FC III/IV at diagnosis, with a significant proportion being in NYHA FC IV $[2,22]$. Survival rates for these patients, even in the modern treatment era, remain poor; in the French Network on Pulmonary Hypertension, for example, estimated survival in incident patients (66\% NYHA FC III and 12.5\% NYHA FC IV) at 1, 2 and 3 years was $85.7 \%, 69.6 \%$ and $54.9 \%$, respectively, and was particularly poor for patients in NYHA FC IV [2].

The potential of upfront dual combination therapy has been investigated in a single randomised controlled study involving epoprostenol and bosentan in patients with severe PAH [14]. Compared with the relatively long-term follow-up of patients in the observational investigations described here and in the dual combination therapy analysis [15], the BREATHE-2 study investigated the efficacy and safety of the combination for 16 weeks. The statistically nonsignificant trend towards an enhanced benefit of the combination regimen over epoprostenol monotherapy provided a rationale for studying upfront combination therapy further, but not enough evidence for this treatment regimen to be adopted in treatment guidelines. However, combination 
therapy where $\mathrm{PAH}$-specific therapy is initiated sequentially is becoming part of normal clinical management of $\mathrm{PAH}$ patients, despite the lack of robust clinical data. The analysis of upfront dual therapy and the current investigation of upfront triple combination therapy provide preliminary evidence that there may be a role for an upfront combination regimen, particularly in patients with severe PAH.

The findings of this study are limited by the small number of patients, who were highly selected in terms of disease severity and aetiology. Moreover, this was not a randomised controlled trial and, as such, these results cannot be directly compared with either a monotherapy approach with epoprostenol or a sequential dual therapy approach with epoprostenol and sildenafil or epoprostenol and bosentan. Large, randomised controlled trials are required to provide further long-term data about the survival/prognosis of patients with severe PAH treated with upfront combination therapy. This study was performed in patients with idiopathic or heritable $\mathrm{PAH}$; further trials will also help physicians to determine the optimum combinations of treatments for different patient cohorts. The findings of this pilot study provide preliminary evidence supporting the long-term benefits of upfront triple combination therapy in patients with severe PAH.

\section{References}

1 Humbert M, Sitbon O, Simonneau G. Treatment of pulmonary arterial hypertension. New Engl J Med 2004; 351: 142-136.

2 Humbert M, Sitbon O, Chaouat A, et al. Survival in patients with idiopathic, familial, and anorexigen-associated pulmonary arterial hypertension in the modern management era. Circulation 2010; 122: 156-163.

3 Galiè N, Hoeper MM, Humbert M, et al. Guidelines for the diagnosis and treatment of pulmonary hypertension. Eur Heart J 2009; 30: 2493-2537.

4 Barst RJ, Rubin LJ, Long WA, et al. A comparison of continuous intravenous epoprostenol (prostacyclin) with conventional therapy for primary pulmonary hypertension. N Engl J Med 1996; 334: 296-301.

5 Sitbon $\mathrm{O}$, Humbert $\mathrm{M}$, Nunes $\mathrm{H}$, et al. Long-term intravenous epoprostenol infusion in primary pulmonary hypertension: prognostic factors and survival. J Am Coll Cardiol 2002; 40: 780-788.

6 McLaughlin VV, Shillington A, Rich S. Survival in primary pulmonary hypertension: the impact of epoprostenol therapy. Circulation 2002; 106: 1477-1482.

7 Nickel N, Golpon H, Greer M, et al. The prognostic impact of follow-up assessments in patients with idiopathic pulmonary arterial hypertension. Eur Respir J 2012; 39: 589-596.

8 McLaughlin VV, Oudiz RJ, Frost A, et al. Randomized study of adding inhaled iloprost to existing bosentan in pulmonary arterial hypertension. Am J Respir Crit Care Med 2006; 174: 1257-1263.

9 McLaughlin VV, Benza RL, Rubin LJ, et al. Addition of inhaled treprostinil to oral therapy for pulmonary arterial hypertension: a randomized controlled clinical trial. J Am Coll Cardiol 2010; 55: 1915-1922.

10 Simonneau G, Rubin LJ, Galiè N, et al. Addition of sildenafil to long-term intravenous epoprostenol therapy in patients with pulmonary arterial hypertension: a randomized trial. Ann Intern Med 2008; 149: 521-530.

11 Hoeper MM, Faulenbach C, Golpon H, et al. Combination therapy with bosentan and sildenafil in idiopathic pulmonary arterial hypertension. Eur Respir J 2004; 24: 1007-1010.

12 Galiè N, Brundage BH, Ghofrani HA, et al. Tadalafil therapy for pulmonary arterial hypertension. Circulation 2009; 119: 2894-2903.

13 Tapson VF, Torres F, Kermeen F, et al. Oral treprostinil for the treatment of pulmonary arterial hypertension in patients on background endothelin receptor antagonist and/or phosphodiesterase type 5 inhibitor therapy (the FREEDOM-C study): a randomized controlled trial. Chest 2012; 142: 1383-1390.

14 Humbert M, Barst RJ, Robbins IM, et al. Combination of bosentan with epoprostenol in pulmonary arterial hypertension: BREATHE-2. Eur Respir J 2004; 24: 353-359.

15 Kemp K, Savale L, O'Callaghan DS, et al. Usefulness of first-line combination therapy with epoprostenol and bosentan in pulmonary arterial hypertension: an observational study. J Heart Lung Transplant 2012; 31: 150-158.

16 Humbert M, Sitbon O, Yaïci A, et al. Survival in incident and prevalent cohorts of patients with pulmonary arterial hypertension. Eur Respir J 2010; 36: 549-555.

17 Sztrymf B, Coulet F, Girerd B, et al. Clinical outcomes of pulmonary arterial hypertension in carriers of BMPR2 mutation. Am J Respir Crit Care Med 2008; 177: 1377-1383.

18 Girerd B, Montani D, Eyries M, et al. Absence of influence of gender and BMPR2 mutation type on clinical phenotypes of pulmonary arterial hypertension. Respir Res 2010; 11: 73.

19 Swiston JR, Johnson SR, Granton JT. Factors that prognosticate mortality in idiopathic pulmonary arterial hypertension: a systematic review of the literature. Respir Med 2010; 104: 1588-1607.

20 Provencher S, Sitbon O, Humbert M, et al. Long-term outcome with first-line bosentan therapy in idiopathic pulmonary arterial hypertension. Eur Heart J 2006; 27: 589-595.

21 Sitbon O, Humbert M, Jaïs X, et al. Long-term response to calcium channel blockers in idiopathic pulmonary arterial hypertension. Circulation 2005; 111: 3105-3111.

22 Humbert M, Sitbon O, Chaouat A, et al. Pulmonary arterial hypertension in France: results from a national registry. Am J Respir Crit Care Med 2006; 173: 1023-1030. 